\title{
Structural insights of mTOR complex 1
}

\author{
Cell Research (2016) 26:267-268. doi:10.1038/cr.2016.10; published online 22 January 2016
}

The mammalian target of rapamycin (mTOR), also known as the mechanistic target of rapamycin, is a central cell growth regulating kinase that forms large molecular complexes in all eukaryotic cells. A paper recently published in Science reports the architecture of $m$ TOR complex 1 (mTORC1) and provides molecular insights into the regulation and substrate selectivity of mTORC1.

The mammalian target of rapamycin (mTOR) exists in two different complexes, mTORC1 and mTORC2, which are distinguished by unique accessory protein Raptor and Rictor, respectively [1]. Rapamycin is an mTORC1-specific inhibitor, which complexes with the FK506-binding $12 \mathrm{kDa}$ protein (FKBP12) and inhibits Raptor-bound, but not Rictor-bound, mTOR. Rapamycin analogs have been used clinically to treat a number of human diseases, including cancer [2]. A wide range of both extra- and intracellular signals, including growth factors, nutrient status and stress conditions, have been shown to regulate mTORC1 to control cell growth. Most notably, mTORC1 is hyperactivated by oncogenic PI3K-Akt signaling and promotes tumor growth [1].

mTORC1 promotes cell growth through phosphorylation of a large number of cellular proteins, including the ribosomal S6 kinase 1 (S6K1) and eIF-4E-binding protein 1 (4E-BP1) [3]. Although mTORC2 shares the same catalytic kinase subunit with mTORC1, it phosphorylates substrates very different from those of mTORC 1 and thus exerts different cellular functions. Despite the extensive studies, the mechanistic understanding of mTORC1 activation and substrate selectivity are rather limited, chiefly due to the lack of three dimensional structure of mTORC1. Understanding of mTORC1 molecular architecture is also of high importance for developing pharmacological drugs to target this pathway.

Cryo-electron microscopy (cryoEM) studies have shown that mTOR forms an obligate dimer with an overall rhomboid shape and a central cavity [4]. However, the reliability of the handedness of the reconstruction and the position of individual subunits was significantly compromised due to the low-resolution (26 A) reconstruction. A subsequent study presented the 3.2 $\AA$ crystal structure of a complex of N-terminally truncated human mTOR and mLST8, which is a subunit commonly present in both mTORC1 and mTORC2. This crystal structure revealed more details of the structure of mTOR kinase domain as well as its inhibition by FKBP12-rapamycin complex [5]. However, information on the subunit arrangement within mTORC1 was missing because only a truncated mTOR fragment was analyzed and the Raptor subunit, which plays a key role in $\mathrm{mTORC} 1$ regulation and substrate selectivity, was lacking.

In a recent paper published in Science, the architecture of human mTORC1 was revealed by high-resolution cryo-EM [6]. The authors purified mTORC1 complex (human mTOR together with Raptor, and mLST8) bound to FKBP12-rapamycin from insect cells. Single particle analysis of cryo-EM yielded a reconstruction with an overall resolution of $5.9 \AA$. To complement the reconstruction of $\mathrm{mTORC} 1$, the authors also resolved the structure of the fungus Chaetomium thermophilum Raptor (CtRaptor), which exhibits 44\% sequence identity to human Raptor. The overall shape of the reconstruction agrees with that observed by low-resolution cyro-EM [4]; however, it appears that the handedness of the previous reconstruction was not assigned correctly. Generally, mTORC1 adopts a cage-like, dimeric architecture and appears in a hollow lozenge shape, in which Raptor and mLST8 contribute peripheral parts of the complex and make up the pinnacles of the longer and shorter axes of the lozenge, respectively. Interestingly, the N-terminus of mTOR, which was not resolved in previous study [5], contains two $\alpha$-helical solenoids. The larger section is a highly curved super-helix, which is named the "horn", while the smaller region adopts a relatively linear arrangement and is referred to as the "bridge" [6]. Both sections are predominantly exposed to the environment, indicating a potential role in binding $\mathrm{mTOR}$ regulators. In addition, the horn and bridge HEAT domains pack against one another, and the first HEAT repeat of the horn region interlocks with the adjacent mTOR FAT domain, through which the two mTOR subunits forms a dimer independent of Raptor [6]. Another interesting observation is that the conformation of the kinase domain appears unaffected by dimerization, suggesting that the regulation of mTORC1 may be mainly through controlling substrate access to the active site.

The authors further investigated how Raptor contributes to the formation of 
A

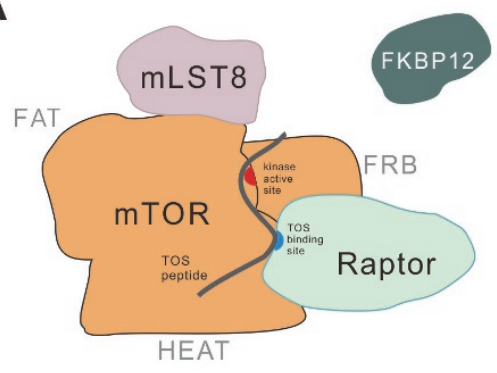

B

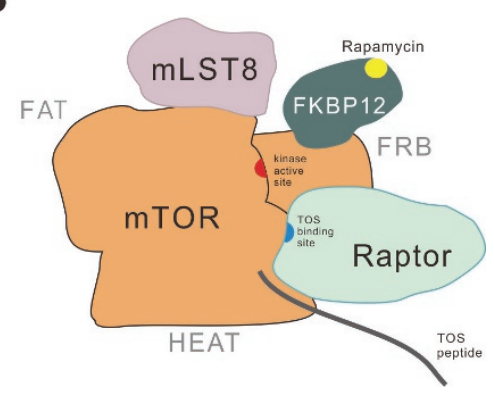

Figure 1 Schematic model of mTORC1 substrate selectivity and delivery. (A) Substrate recruitment is dependent on their TOR signaling (TOS) motif binding to Raptor TOS-binding site. mTOR FRB domain and mLST8 prevent phosphorylation towards non-cognate substrates by limiting the access to the ATP-binding cleft. Raptor binding further restricts the access to the active site, resulting in the enclosure of the active site cleft from all directions. Only when the TOS motif present in the substrate is recognized by Raptor, substrates can be delivered to the mTOR kinase active site and phosphorylated. (B) The FKBP12-rapamycin complex binding to the FRB domain further blocks the active site cleft and prevents the access of substrate to kinase active site.

mTORC1 complex. Raptor interacts with mTOR through an $\alpha$-solenoid stack formed between the horn and bridge domains of mTOR via the Raptor armadillo domain [6]. It is proposed that Raptor stabilizes the N-terminal region of mTOR by providing roughly twothirds of the interaction surface with HEAT domains. As mentioned above, the formation of mTORC1 dimer is dependent on interaction of mTOR domains, but not Raptor, thus a model is suggested that Raptor binding may stabilize mTOR N-terminal conformation without directly engaging in dimer formation.

The structure of mTORC1 also provides implications of mTORC1 substrate selectivity and delivery. Previous report has revealed that mTOR FRB domain and mLST8 prevent activity toward non-cognate substrates by limit- ing access to the ATP-binding cleft [5]. According to the current architecture, Raptor binding further restricts the access to the active site, resulting in the enclosure of the active site cleft from all directions and reduction of its width to $20 \AA$ [6]. Moreover, binding of FKBP12-rapamycin complex to the FRB domain of mTORC1 further reduces the active site cleft to $\sim 10 \AA[6]$. Taken together, this model shows how architectural subunits of mTORC1 and FKBP12-rapamycin limit access to the recessed mTOR active site (Figure 1). It is notable that in contrary to previous findings [4], this study indicates that FKBP12-rapamycin binding has no effect on mTORC1 stability.

In summary, the new structure reveals insights into the mTORC1 architecture and important clues for mTORC1 functional regulation. The kinase domain of mTOR maintains a constitutively active conformation at all times. Association with Raptor limits substrate accessibility to the mTOR kinase active site as the Raptor RNC domain is positioned directly at the mTOR active site cleft, thereby explaining how Raptor modulates substrate selectivity of mTORC1. Furthermore, the new structure explains mTORC1 inhibition by FKBP12-rapamycin through blocking substrate accessibility to the mTOR kinase active site. It should be noted that the architecture of mTORC1 still needs further improvement as the current resolution $(5.9 \AA)$ is not sufficient to reveal amino acid side chains of subunits and critical sites for dimer formation and activity control.

Hai-Xin Yuan ${ }^{1}$, Kun-Liang Guan ${ }^{2}$

${ }^{1}$ Molecular Cell Biology Laboratory, Institutes of Biomedical Sciences, Shanghai Medical College, Fudan University, Shanghai 200032, China; ${ }^{2}$ Department of Pharmacology and Moores Cancer Center, University of California, San Diego, La Jolla, CA 92093, USA

Correspondence: Hai-Xin Yuan ${ }^{\text {a }}$,

Kun-Liang Guan ${ }^{\mathrm{b}}$

${ }^{a}$ E-mail: yuanhaixin@fudan.edu.cn

bE-mail: kuguan@ucsd.edu

\section{References}

1 Laplante M, Sabatini DM. Cell 2012; 149:274-293

2 Benjamin D, Colombi M, Moroni C, et al. Nat Rev Drug Discov 2011; 10:868-880.

3 Ma XM, Blenis J. Nat Rev Mol Cell Bio 2009; 10:307-318.

4 Yip CK, Murata K, Walz T, et al. Mol Cell 2010; 38:768-774.

5 Yang H, Rudge DG, Koos JD, et al. Nature 2013; 497:217-223.

6 Aylett CH, Sauer E, Imseng S, et al. Science 2016; 351:48-52. 